\title{
Passivity based force control of hydraulic robots
}

\author{
Satoru Sakai* Stefano Stramigioli** \\ * Chiba University (e-mail: satorusakai@faculty.chiba-u.jp) \\ ** Twente University
}

\begin{abstract}
This paper gives a new nonlinear force control of hydraulic robots. Unlike the electrical robots, hydraulic robots are governed not only by the energy conservation but also by the mass conservation. First we propose a generalized continuity law and a canonical form of hydraulic robots. Second, we propose a new nonlinear robust force control based on passivity in order to achieve the desired force in the presence of unknown environment consisting of the energy storing elements and dissipative elements. Finally, we confirm the validity of our proposed methods by simulation and experiment.
\end{abstract}

Keywords: Passivity based control, Casimir generation, robotic manipulators

\section{INTRODUCTION}

This paper gives a new design method of force control for hydraulic manipulators in port-Hamiltonian form (van der Schaft (2000)). Port-Hamiltonian systems are generalization of Hamiltonian systems in classical mechanics and can model many systems such as electro-mechanical systems, mechanical systems with nonholonomic constraints (Maschke and van der Schaft (1994)), distributed systems and their mixed systems (Macchelli and Melchiorri (2005)) as well as classical mechanical systems.

Passivity is one of the most important properties of portHamiltonian systems and some passivity based control methods, originally from Takegaki and Arimoto (1981), were developed, such as, Energy-Casimir methods (van der Schaft (2000)), the generalized canonical transformations (Fujimoto and Sugie (2001)), IDA-PBC (Ortega and Garcia-Canseco (2004)) and IPC approach (Stramigioli et al. (1998)) and so on.

Modeling and control of several fluid systems are discussed in port-Hamiltonian form. For example, Ramkrishna and van. der. Schaft (2006) discuss infinite dimensional canal systems in three dimensional space and Johanson (2006) discuss the four-tank systems based on IDA-PBC. These fluid systems have free-surface and are incompressible. Riccardo et al. (2006) discuss the modeling of hydraulic arms and show some experimental results. Gernot and Schlacher (2005) discuss the control of the hydraulic arms in portHamiltonian form. In these approaches, the modeling is based on the standard procedure in port-Hamiltonian framework and the positing control is discussed in almost all cases. Apart from these approaches, we discuss the modeling and force control of the class of fluid mechanical systems, such as hydraulic robot arms, pneumatic robot arms, based on the other structural property.

This paper is organized as follows. In Section 2, we refer port-Hamiltonian systems and their properties, especially Casimir functions and hydraulic systems. In Section 3, we discuss the modeling and the canonical form of the hydraulic robots. In Section 4, we give a new passivity based force control of hydraulic mechanical systems. In Section 5 , the proposed method are confirmed by simulation and in Section 6 , by experiment. Finally we conclude this paper in Section 7.

In this paper, $I_{n}$ is $n \times n$ identity matrix, $\mathbf{R}^{m \times n}$ is the real space of $m$ rows and $n$ columns matrix.

\section{PRELIMINARY}

In this section, we refer basic concepts such as passivity, port-Hamiltonian systems and their control methods.

\subsection{A Special Class of Port-Hamiltonian systems}

Definition 1 A (simplified version of) port-Hamiltonian system with a Hamiltonian $H(x) \in \mathbf{R}$ is a system described by

$$
\left\{\begin{array}{l}
\dot{x}=J(x) \frac{\partial H(x)}{\partial x}^{\mathrm{T}}+g(x) u \\
y=g(x)^{\mathrm{T}} \frac{\partial H(x)}{\partial x}^{\mathrm{T}}
\end{array}\right.
$$

with $u, y \in \mathbf{R}^{m}, x \in \mathbf{R}^{n}$ and a skew symmetric matrix $J(x)$, i.e. $-J(x)=J(x)^{\mathrm{T}}$ holds. The following property of such systems is known.

Lemma 1 Consider the port-Hamiltonian system (1). Suppose the Hamiltonian $H(x)$ satisfies $H(x) \geq H(0)=0$. Then the input-output mapping $u \mapsto y$ of the system is passive with respect to the storage function $H$, and the feedback

$$
u=-D(x) y
$$

with a matrix $D(x)>0 \in \mathbf{R}^{m \times m}$ renders $(u, y) \rightarrow 0$. Furthermore if $H(x)$ is positive definite and if the system is zero-state detectable, then the feedback (2) renders the origin asymptotically stable. 


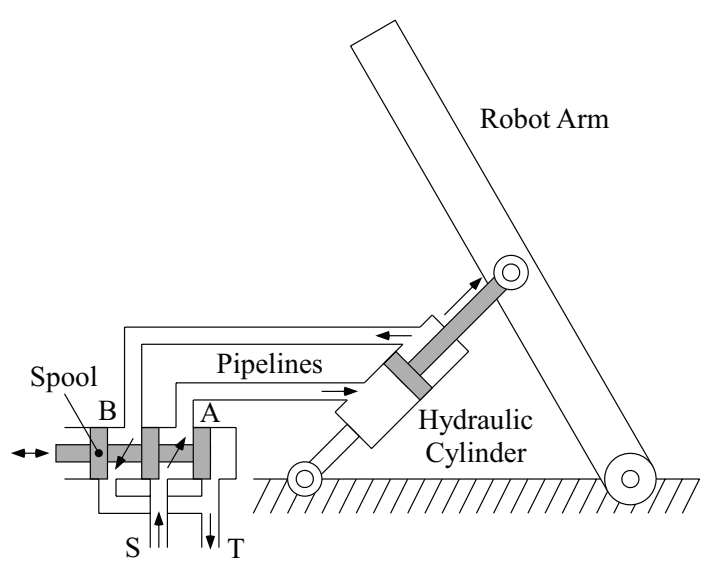

Fig. 1. Hydraulic systems

\subsection{Casimir functions}

One of other properties of port-Hamiltonian systems are the existence of Casimir functions. Casimir functions (with respect to $J$ ) are defined as solutions of the following PDE,

$$
\frac{\partial C(x)}{\partial x} J(x) \equiv 0
$$

Not only Hamiltonian, but also Casimir functions are the special first integrals. However Casimir functions are different from Hamiltonian and

$$
\dot{C} \equiv 0
$$

holds for any Hamiltonian $H(x)$ when $u=0$. Unlike Hamiltonian, Casimir functions are not bounded from below nor upper in general. Casimir functions do not alway exist for port-Hamiltonian systems in general, but exist in some important cases in robotics.

\subsection{Hydraulic robots}

Fig.1 shows hydraulic arms. Hydraulic arms are different from electrical arms with respect to the complexity in the actuators. Especially, the output signals from the actuators are not only the input into the mechanical systems but also the input into the actuators as the internal loop.

While the dynamics of the electrical arms can be derived from the viewpoint of the energy conservations, the dynamics of the hydraulic arms can be derived from the viewpoint of the mass conservations as

$$
\frac{V}{b} \dot{p}=Q_{2}-Q_{1}
$$

where $p$ is the pressure, $V$ is the volume, $\beta$ is the bulk modulus and $Q_{i}$ are modeled as

$$
Q_{i}=k v\left(p, \operatorname{sgn}\left(x_{v}\right)\right) x_{v}=k \operatorname{sgn}\left(x_{v}\right) \sqrt{\left|p-p_{s}\right|} x_{v}
$$

with the spool displacement $x_{v}$, the supply pressure $p_{s}$ and the spool coefficient $k$.

\section{NONLINEAR MODELING}

A nonlinear modeling of hydraulic robot arms are discussed in this section. First, the (electrical) manipulators are described in the port-Hamiltonian form

$$
\Sigma_{m}:\left\{\begin{array}{l}
{\left[\begin{array}{l}
\dot{q_{m}} \\
\dot{p}_{m}
\end{array}\right]=\left[\begin{array}{cc}
0 & I \\
-I-C_{m}
\end{array}\right]\left[\begin{array}{l}
\frac{\partial H_{m}}{\partial q_{m}} \\
\frac{\partial H_{m}}{\partial p_{m}} \mathrm{~T}
\end{array}\right]+\left[\begin{array}{c}
0 \\
G_{m}
\end{array}\right] u_{m}} \\
y_{m}=G_{m}^{\mathrm{T}}{\frac{\partial H_{m}}{\partial p_{m}}}^{\mathrm{T}}
\end{array}\right.
$$

where $q_{m}$ and $p_{m}$ are the position and the momentum, $M\left(q_{m}\right)=M\left(q_{m}\right)^{\mathrm{T}}>0, U\left(q_{m}\right) \geq 0$, Hamiltonian $H_{m}=$ $(1 / 2)\left(p^{\mathrm{T}} M^{-1} p\right)+U\left(q_{m}\right)$ and the nonsingular matrix $G_{m}$.

Definition 1 (Generalized continuity law) An inputoutput system

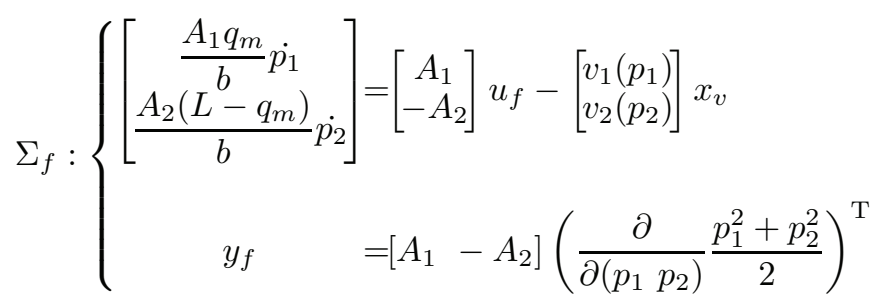

is the generalized continuity law where $u_{f}, x_{v} \in \mathbf{R}$ are the inputs, $y_{f} \in \mathbf{R}$ is the output and $v_{i}$ is Bernoulli velocity $A_{i}$ is the cylinder area, $q_{m} \in[0, L]=B$ (Fig.2).

This is a generalization of the mass conservation as a portHamiltonian system is the generalization of the energy conservation. Note that equation (8) is not written in the form (1).

Theorem 1 (Canonical form of hydraulic robots) Consider the system $\Sigma_{f}$ and the system $\Sigma_{m}$. Then there exists a coordinate transformation and a scaler function $H_{f m}(x)$ such that the following feedback interconnection

$$
\left\{\begin{array}{l}
u_{f}=-y_{m} \\
u_{m}=+y_{f}
\end{array}\right.
$$

gives the closed-loop systems

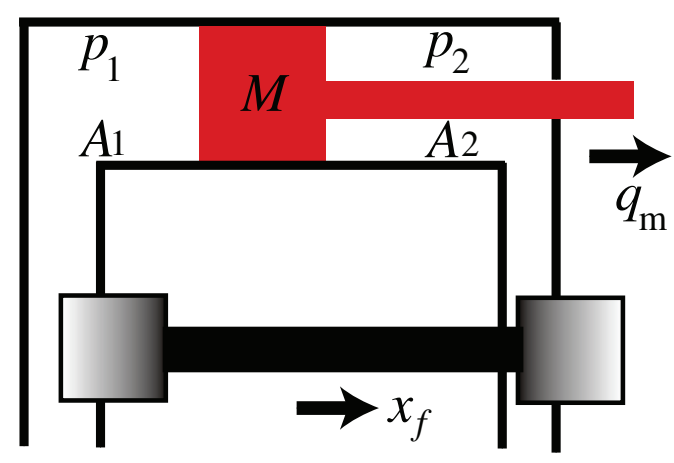

Fig. 2. Equivalent model 


$$
\Sigma_{f m}:\left\{\begin{array}{l}
{\left[\begin{array}{c}
q_{m} \\
p_{m} \\
\dot{C}_{1} \\
\dot{C}_{2}
\end{array}\right]=\underbrace{\left[\begin{array}{cc}
J_{s} & 0 \\
0 & 0
\end{array}\right]}_{\equiv J}\left[\begin{array}{c}
\frac{\partial H_{f m}}{\partial\left(q_{m}, p_{m}\right)} \\
\frac{\partial H_{f m}}{\partial\left(C_{1}, C_{2}\right)}
\end{array}\right]+\underbrace{\left[\begin{array}{c}
0 \\
0 \\
\frac{b v_{1}}{A_{1} q_{m}} \\
\frac{b v_{2}}{A_{2}\left(L-q_{m}\right)}
\end{array}\right]}_{\equiv g_{f m}}} \\
y_{v}=g_{f m}^{\mathrm{T}} \frac{\partial H_{f m}}{\partial\left(q_{m}, p_{m}, C_{1}, C_{2}\right)}
\end{array}\right.
$$

with $x=\left(q_{m}, p_{m}, C_{1}, C_{2}\right) \in \mathbf{R}^{4}$ and $J_{s}=\left[\begin{array}{cc}0 & 1 \\ -1 & 0\end{array}\right]$.

\section{Proof of Theorem 1}

Casimir function $C_{1}, C_{2}$ as the solutions of PDE (3) gives the following transformation

$$
\left[\begin{array}{c}
q_{m} \\
p_{m} \\
C_{1} \\
C_{2}
\end{array}\right]=\phi\left(q_{m}, p_{m}, p_{1}, p_{2}\right)=\left[\begin{array}{c}
q_{m} \\
p_{m} \\
p_{1}+\log \left(q_{m}\right) \\
p_{2}-\log \left(L-q_{m}\right)
\end{array}\right]
$$

where $\frac{\partial \phi}{\partial x}$ is invertible. This implies the feedback interconnection (9) gives the port-Hamiltonian systems (12) with a Hamiltonian

$$
\begin{aligned}
& H_{f m}=H_{m}+A_{1}\left(b \left(e^{C_{1} / b}-q_{m}+\right.\right. \\
& \left.\left.q_{m} \log \left(q_{m}\right)\right)-q_{m} C_{1}\right)+A_{2}\left(b \left(e^{C_{2} / b}-\left(L-q_{m}\right)+\right.\right. \\
& \left.\left.\left(L-q_{m}\right) \log \left(L-q_{m}\right)\right)-\left(L-q_{m}\right) C_{2}\right) .
\end{aligned}
$$

(Q.E.D.)

Remark 1 The above canonical form makes the dynamics of $C_{1}, C_{2}$ a drift-less system, that is, the hydraulic arms is a serial connection of a driving system and a driven system as the electrical arms. This fact is important with respect to the simplicity in the control systems design/analysis.

Remark 2 The friction effects (Coulomb effects, Stribeck effects) are easily modeled in the above situation but omitted here for the space limitation.

\section{NONLINEAR FORCE CONTROL}

In this section, a new force control of hydraulic arms are given in the presence of unknown environment.

Theorem 2(Nonlinear force control) Consider the unknown environment

$$
\Sigma_{e}:\left\{\begin{array}{l}
\dot{q_{m}}=y_{m} \\
F_{e}=-\frac{\partial U_{e}}{\partial q_{m}}-C_{e} y_{m}
\end{array}\right.
$$

and the (modified) system $\Sigma_{f m}$

$$
\Sigma_{f m}:\left\{\begin{array}{c}
{\left[\begin{array}{c}
\dot{q}_{m} \\
\dot{p}_{m} \\
\dot{C}_{1} \\
\dot{C}_{2}
\end{array}\right]=\left[\begin{array}{ll}
J_{s} 0 \\
0 & 0
\end{array}\right]\left[\begin{array}{c}
\frac{\partial H_{f m}}{\partial\left(q_{m}, p_{m}\right)} \\
\frac{\partial H_{f m}}{\partial\left(C_{1}, C_{2}\right)}
\end{array}\right]+\left[\begin{array}{cc}
0 & 0 \\
0 & 1 \\
\frac{b v_{1}}{A_{1} q_{m}} & 0 \\
\frac{b v_{2}}{A_{2}\left(L-q_{m}\right)} & 0
\end{array}\right]\left[\begin{array}{l}
x_{v} \\
F_{e}
\end{array}\right]} \\
y_{v}=g_{f m}^{\mathrm{T}} \frac{\partial H_{f m}}{\partial\left(q_{m}, p_{m}, C_{1}, C_{2}\right)}
\end{array}\right.
$$

and assume that $\frac{\partial U_{e}(q)}{\partial q}=F_{d}$ has a solution $q_{m}^{*} \in \mathbf{R}$. Then the force controller

$$
\Sigma_{F}: x_{v}=-\left(A_{1} g_{3}-A_{2} g_{4}\right) R\left(F-F_{d}\right)
$$

makes the set $\Omega=\left\{F=F_{d}\right\}$ asymptotically stable where $R>0$ is the gain, $F_{d}$ is the desired force, $U_{e}, C_{e}$ are the unknown parameters of the environment and $g_{3}, g_{4}$ are the third and the fourth elements of $g_{f m}$.

\section{Proof of Theorem 2}

A coordinate transformation

$$
\left[\begin{array}{c}
q_{m} \\
p_{m} \\
F \\
F^{\perp}
\end{array}\right]=\left[\begin{array}{c}
q_{m} \\
p_{m} \\
A_{1} p_{1}-A_{2} p_{2} \\
p_{1} /\left(2 A_{2}\right)+p_{2} /\left(2 A_{1}\right)
\end{array}\right]
$$

is invertible and the system $\Sigma_{f m}$ is converted into

$$
\left[\begin{array}{c}
q_{m} \\
p_{m} \\
\dot{F} \\
\dot{F} \perp
\end{array}\right]=\left[\begin{array}{cccc}
0 & 1 & 0 & 0 \\
-1 & 0 & J_{23} & J_{24} \\
0 & -J_{23} & 0 & 0 \\
0 & -J_{24} & 0 & 0
\end{array}\right]\left[\begin{array}{l}
\frac{\partial H_{f m}}{\partial q_{m}}{ }^{\mathrm{T}} \\
\frac{\partial H_{f m}}{\partial p_{m}} \\
\frac{\partial H_{f m}}{\partial F} \\
\frac{\partial H_{f m}}{\partial F^{\perp}}
\end{array}\right]+\left[\begin{array}{c}
0 \\
0 \\
g_{a} \\
g_{b}
\end{array}\right] x_{v}
$$

where

$$
\left\{\begin{array}{l}
J_{23}=b\left(A_{1}^{2}+A_{2}^{2}\right) / V \\
J_{24}=b\left(A_{1}^{2}-A_{2}^{2}\right) /\left(2 V A_{1} A_{2}\right) \\
g_{a}=A_{1} g_{3}-A_{2} g_{4}(\geq 0) \\
g_{b}=g_{3} /\left(2 A_{2}\right)+g_{4} /\left(2 A_{1}\right)
\end{array}\right.
$$

and the identity

$$
J_{23}{\frac{\partial H_{f m}}{\partial F}}^{\mathrm{T}}+J_{24}{\frac{\partial H_{f m}}{\partial F^{\perp}}}^{\mathrm{T}}-F=0
$$

holds. By separating the dynamics of $F^{\perp}$, the reduced system of $q_{m}, p_{m}, F$ is

$$
\Sigma_{f m r}:\left\{\left[\begin{array}{c}
\dot{q_{m}} \\
p_{m}^{\cdot} \\
\dot{F}
\end{array}\right]=\left[\begin{array}{ccc}
0 & 1 & 0 \\
-1 & 0 & J_{23} \\
0 & -J_{23} & 0
\end{array}\right]\left[\begin{array}{l}
\frac{\partial H_{f m}}{\partial q_{m}} \\
\frac{\partial H_{f m}}{\partial p_{m}} \\
\frac{\partial H_{f m}}{\partial F}
\end{array}\right]+\left[\begin{array}{c}
0 \\
0 \\
g_{a}(t)
\end{array}\right] x_{v}\right.
$$

where $g_{a}(t)=g_{a}\left(F, F^{\perp}\right)$. From $q_{m} \in B$, the function

$$
H_{c l}=H_{f m}+F_{d}\left(L-q_{m}\right)
$$

is lower bounded and the coordinate transformation 


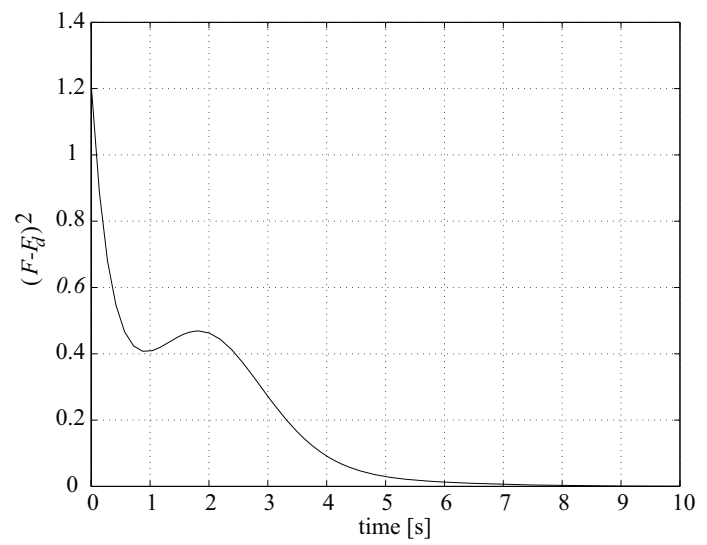

Fig. 3. Response of $\left(F-F_{d}\right)^{2}$

$$
\left[\begin{array}{c}
q_{m} \\
p_{m} \\
\tilde{F}_{A}
\end{array}\right]=\left[\begin{array}{ccc}
1 & 0 & 0 \\
0 & 1 & 0 \\
0 & 0 & J_{23}^{-1}
\end{array}\right]\left[\begin{array}{c}
q_{m} \\
p_{m} \\
F-F_{d}
\end{array}\right]
$$

makes the closed-loop system (including the environment)

$$
\left[\begin{array}{c}
q_{m} \\
\dot{p}_{m} \\
\dot{\tilde{F}}_{A}
\end{array}\right]=\left[\begin{array}{ccc}
0 & 1 & 0 \\
-1-C_{m}-C_{e} & +1 \\
0 & -1 & -R g_{a}^{2}
\end{array}\right]\left[\begin{array}{l}
\frac{\partial H_{c l}+U_{e}}{\partial q_{m}} \\
\frac{\partial H_{c l}+U_{e}}{\partial p_{m}} \\
\frac{\partial H_{c l}+U_{e}}{\partial \tilde{F}_{A}}
\end{array}\right]
$$

This implies

$$
\dot{H}_{c l}+\dot{U}_{e} \leq-C_{m}\left(\frac{\partial H_{c l}}{\partial p_{m}}\right)^{2}-R g_{a}^{2}\left(\frac{\partial H_{c l}}{\partial \tilde{F}_{A}}\right)^{2}
$$

is non-positive and is the sum of squares with respect to $p_{m}, \tilde{F}_{A}$ and $\Omega=\left\{p_{m}=\tilde{F}_{A}=0\right\}$ is a positive-invariant set. Since we have

$$
\dot{p}_{m}=-\left(-F_{d}+\frac{\partial U_{e}}{\partial q_{m}}\right)+J_{23} \tilde{F}_{A},
$$

the assumption and $\overrightarrow{p_{m}} \rightarrow 0$ means the convergence $F \rightarrow F_{d}$ and

$$
q_{m} \rightarrow q_{m}^{*} \text { s.t. } \frac{\partial U_{e}\left(q_{m}\right)}{\partial q_{m}}=F_{d}
$$

holds. (Q.E.D.)

Note that the closed-loop system is robust against the parameter perturbation as long as the inequality (20) holds. It is possible to improve the transient behavior by adjusting the gain $R$.

\section{SIMULATION}

A simple mechanical system is considered in this section. This system is one dof and interacts with unknown energy storing element $\left(U\left(q_{m}\right)=(1 / 2) k_{e} q_{m}^{2}\right)$ and unknown energy dissipative element. The desired force is $F_{d}=1$ and the other parameters $V, b$ and the gain $D$ are normalized. The initial conditions are $q_{m}(0)=-1, p_{m}(0)=1$, $\tilde{F}_{A}(0)=1.2$.

Fig.3 shows the time response of $\left(F-F_{d}\right)^{2}$. It is confirmed that the cylinder force $F$ converts to the desired

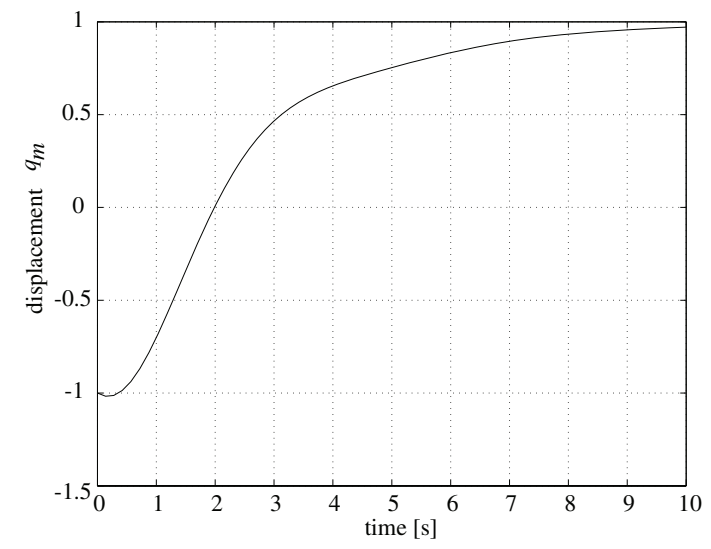

Fig. 4. Response of $q_{m}$

$F_{d}$ smoothly against the environment with unknown parameters $k_{e}, C_{e}$. Fig.4 shows the time response of $q_{m}$. The behavior of $q_{m}$ depends on the unknown parameters $k_{e}, C_{e}$ and converts to a value $q_{m}^{*}$ asymptotically. In all, the validity of the proposed controller is confirmed in the simulation phase.

\section{EXPERIMENT}

\subsection{Experimental setup}

Fig.5 shows a standard hydraulic robot. According to the actual applications, this robots has not only rotational joints but also the translational joints. This robot is reconfigurable, that is, has nine configurations. This is important because the nonlinear effect (the gravity, the centrifugal, Coriolis) can be deleted in the experiments. Table 2 shows the specification.

Table 1. Specification of robot arm

\begin{tabular}{l|l}
\hline parameters & values \\
\hline length of link & $0.5 \mathrm{~m}$ \\
cylinder area $A_{1}$ & $7.010^{-4} \mathrm{~m}^{2}$ \\
cylinder area $A_{2}$ & $5.410^{-4} \mathrm{~m}^{2}$ \\
cylinder stroke $L$ & $0.75 \mathrm{~m}$ \\
mass & $40 \mathrm{~kg}$ \\
viscous $C_{m}$ & $9500 \mathrm{Ns} / \mathrm{m}$ \\
bulk modulus $b$ & $1.510^{9} \mathrm{~Pa}$ \\
spool coefficient $k$ & $2.010^{-7} \mathrm{~m}^{3} /(\sqrt{\mathrm{Pa}} \mathrm{Vs})$ \\
pump pressure & $7.0 \mathrm{MPa}$ \\
\hline
\end{tabular}

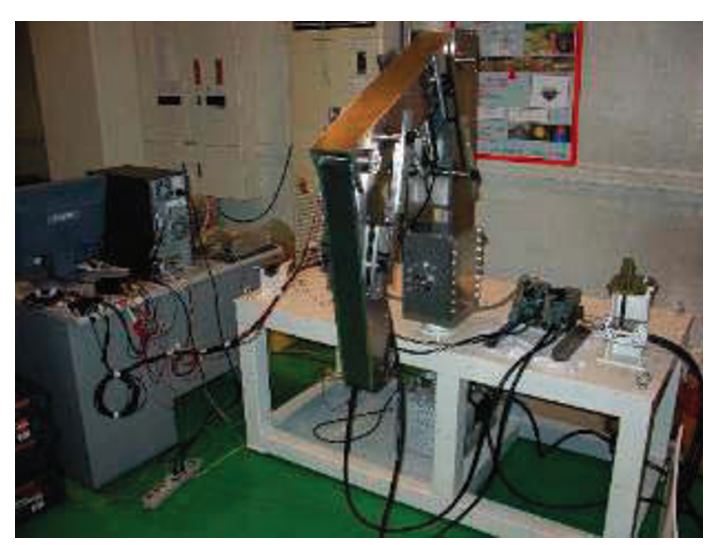

Fig. 5. Standard hydraulic arm 


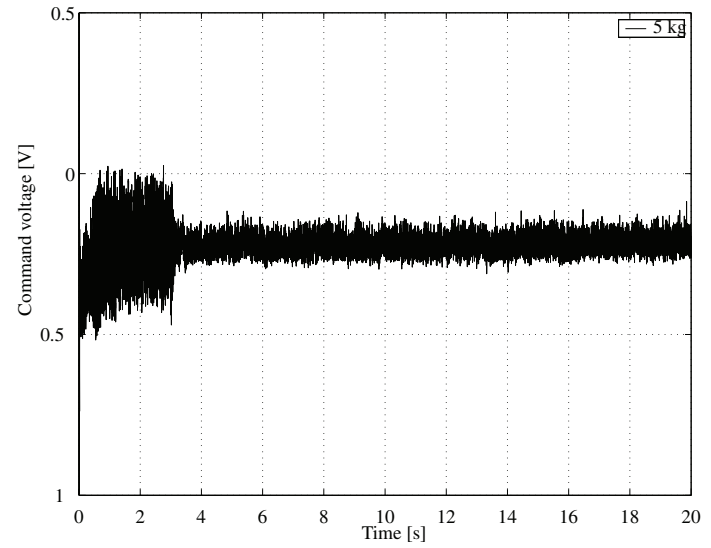

Fig. 6. Response of the command $\left(F_{d}=5\right)$

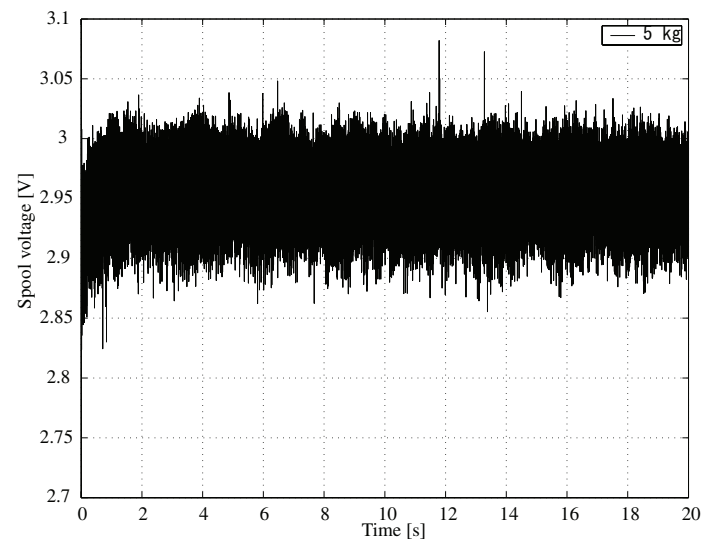

Fig. 7. Response of the spool displacement $\left(F_{d}=5\right)$

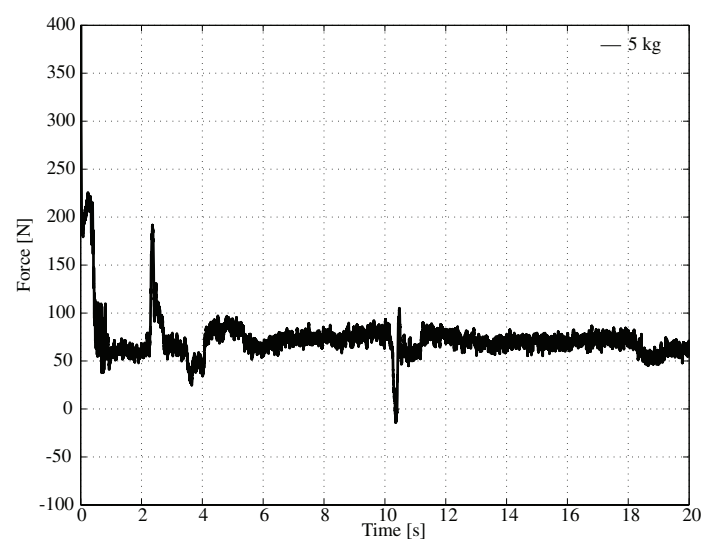

Fig. 8. Response of the cylinder force $\left(F_{d}=5\right)$

\subsection{Control experimental result and discussion}

The control experiment is executed in the Cartesian configuration and make the environment parameters $U_{e}=$ $m_{e} g q_{m}, C_{e}=0\left(m_{e}=5 \mathrm{~kg}, 15 \mathrm{~kg}\right) .300 \mathrm{~Hz}$ dizzier signals are added on the reference signal. The spool displacement is measured by LVDT.

Fig.6 shows the reference signal in the case of $F_{d}=5$ and Fig.7-9 show the spool displacement $x_{v}$, the cylinder force $F$, and the cylinder displacement $q_{m}$. The cylinder force $F$ converts to the desired value and keeps the position. The behavior of the force $F$ has become smaller after the settling time while the behavior of the cylinder $q_{m}$

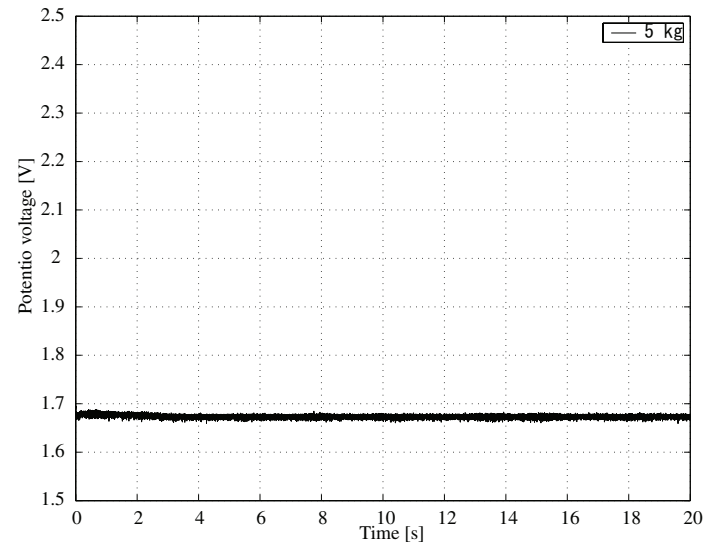

Fig. 9. Response of the cylinder displacement $\left(F_{d}=5\right)$

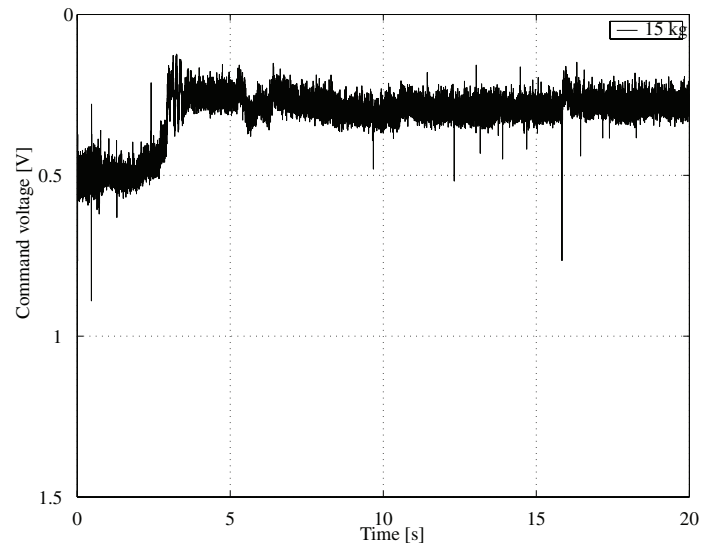

Fig. 10. Response of the command $\left(F_{d}=15\right)$

is not large. Fig.10, 11, 12, 13 are the responses in the case of $F_{d}=15$. The force $F$ converts to the desired value and the transient behaviors are almost similar to those in the case of $F_{d}=5$. Note that the gain $R$ is any positive value and the controller does not require the hydraulic system parameters and the structure such as Bernoulli velocity. Indeed the force signal is asymptotically stable against the complexity in the actual system. The robustness is confirmed from the experiment.

\section{CONCLUSIONS}

A passivity based force control for hydraulic robots are discussed in this paper. The generalized continuity law is defined and a canonical form of hydraulic robots is derived. In the presence of the unknown environment, a robust force controller is proposed and the validity is confirmed by the simulation and the experiment.

\section{REFERENCES}

K. Fujimoto and T. Sugie. Canonical transformation and stabilization of generalized hamiltonian systems. Systems \& Control Letters, 42(3):217-227, 2001.

G. Gernot and K Schlacher. Energy-based nonlinear control of hydraulically actuated mechanical systems. In Proc. of the 44th Conference on Decision and Control, pages 7520-7525, 2005.

K.H. Johanson. Interconnection and damping assigment passivity-based control of a four-tank system. In Proc. 


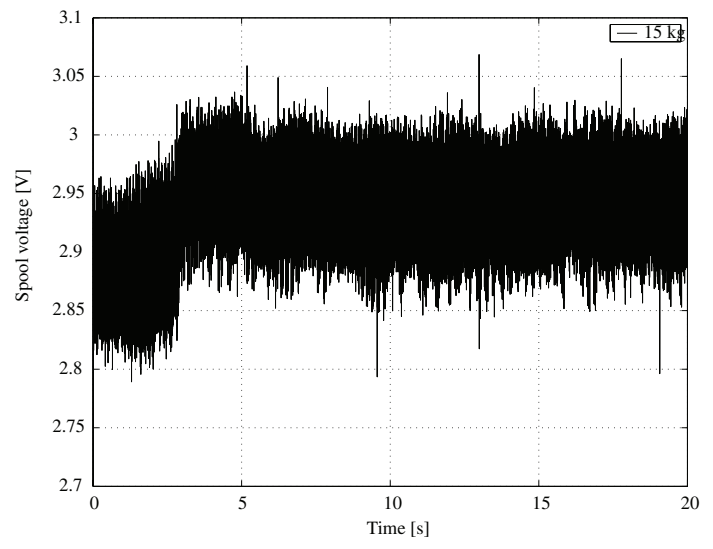

Fig. 11. Response of the spool displacement $\left(F_{d}=15\right)$

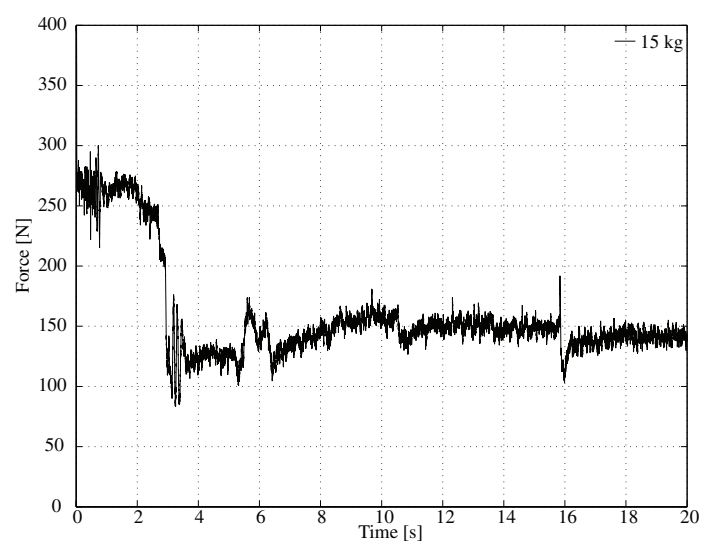

Fig. 12. Response the cylinder force $\left(F_{d}=15\right)$

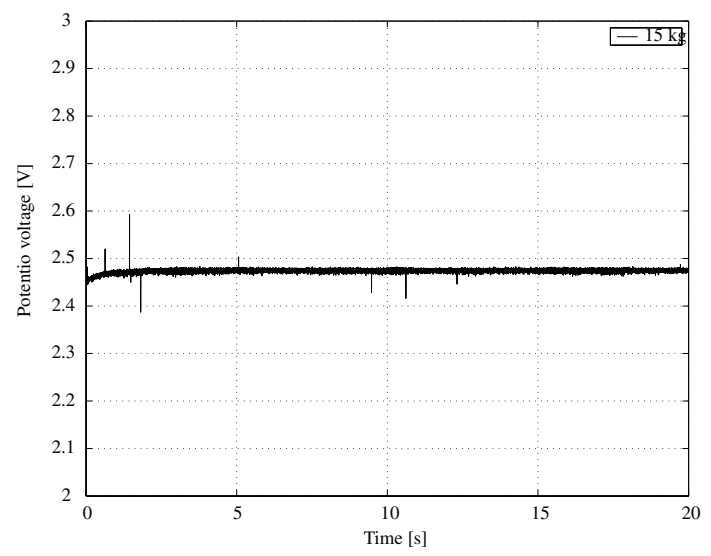

Fig. 13. Response of the cylinder force $\left(F_{d}=15\right)$

of 3rd IFAC workshop on Lagrangian and Hamiltonian systems and control, pages 69-74, 2006.

A. Macchelli and C. Melchiorri. Control by interconnection of mixed port hamiltonian systems. IEEE Trans. Automatic Control, 50(11):1839-1844, 2005.

B. M. J. Maschke and A. J. van der Schaft. A Hamiltonian approach to stabilization of nonholonomic mechanical systems. In Proc. 33rd IEEE Conf. on Decision and Control, pages 2950-2954, 1994.

R. Ortega and Eloisa Garcia-Canseco. Interconection and damping assignment passivity-based control: A survey. European Journal of Control, pages 1-27, 2004.
P. Ramkrishna and Arjan van. der. Schaft. A porthamiltonian approach to modeling and interconnections of canal systems. In Network modeling and control of physical systems, page WeA08, 2006.

M. Riccardo, Z. Roberto, and F. Paolo. Dynamic model of an electro-hydraulic three point hitch. In Proc. of the 2006 Amecican Control Conference, pages 1868-1873, 2006.

S. Stramigioli, B. M. J. Maschke, and A. J. van der Schaft. Passive output feedback and port interconnection. In Proc. 4th IFAC Symp. Nonlinear Control Systems, pages 613-618, 1998.

M. Takegaki and S. Arimoto. A new feedback method for dynamic control of manipulators. Trans. ASME, J. Dyn. Syst., Meas., Control, 103:119-125, 1981.

A. J. van der Schaft. $L_{2}$-Gain and Passivity Techniques in Nonlinear Control. Springer-Verlag, London, 2000. 